\title{
Heterogeneous and Competitive Multiagent Networks: Couple-Group Consensus with Communication or Input Time Delays
}

\author{
Nanxiang Yu, ${ }^{1,2}$ Lianghao Ji, ${ }^{1}$ and Fengmin $\mathrm{Yu}^{2}$ \\ ${ }^{1}$ Chongqing Key Laboratory of Computational Intelligence, Chongqing University of Posts and Telecommunications, \\ Chongqing 400065, China \\ ${ }^{2}$ School of Mathematics and Physics, Chongqing University of Posts and Telecommunications, Chongqing 400065, China \\ Correspondence should be addressed to Lianghao Ji; lianghao.ji@gmail.com
}

Received 14 April 2017; Revised 3 July 2017; Accepted 22 November 2017; Published 13 December 2017

Academic Editor: Sigurdur F. Hafstein

Copyright (C) 2017 Nanxiang Yu et al. This is an open access article distributed under the Creative Commons Attribution License, which permits unrestricted use, distribution, and reproduction in any medium, provided the original work is properly cited.

\begin{abstract}
This paper discusses the couple-group consensus problems for a class of heterogeneous multiagent networks including the following two cases: with communication and input time delays, respectively. Different from the related cooperative networks, two novel delayed group consensus protocols are designed based on the competitive relationship between the agents. Furthermore, we absolutely relax the in-degree balance and other restrictive preconditions which existed in the relevant works. Some sufficient algebraic criteria for the achievement of couple-group consensus and the upper bound of the input time delays are technically obtained via the frequency domain method and matrix theory, respectively. The results show that the achievement of the couple-group consensus depends on the second-order agents' in-degree and the control parameters of the systems, whereas it is independent of the communication time delays. Meanwhile, the upper bound of the input time delay is determined by the control parameters and the in-degree of the first-order agents. Finally, the validity of the proposed results is verified by several simulated examples.
\end{abstract}

\section{Introduction}

Consensus is a typical problem of coordinate control of complex multiagent systems (MASs). It means that all the agents will be in agreement with each other as time goes on. Group (cluster) consensus is an extended issue of consensus problem. To MASs, the achievement of group consensus implies that consensus can be reached in each subgroup, respectively. In the last decades, consensus and group consensus problems have attracted the attention of the researchers in many fields due to their extensive applications. Such as in mobile robot systems [1,2], smart grid [3], pinning control, and optimization of complex systems [4-9].

Up to now, much progress has been made in the study of consensus and group consensus issues. However, with the increasing demand for application, there are still many problems that need to be faced. Such as most of the related works focus on the homogeneous MASs. In fact, complex systems are often heterogeneous in the field of practical engineering. Namely, the dynamics of the agents are different, at least not identical. Hence, more and more attention should be paid on heterogeneous MASs. Recently, lots of interesting works about heterogeneous MASs have been reported. For example, in $[10,11]$, the authors discussed the synchronization of the heterogeneous systems and obtained some sufficient and/or necessary criteria which can guarantee the achievement of synchronization. In $[12,13]$, to the heterogeneous systems with undirected topology, the consensus and the globally bounded consensus problems were studied, respectively. In [14-16], the authors discussed the synchronization and consensus issues for heterogeneous systems with the influence of time delays, respectively. Meanwhile, the consensus problems for the discrete-time heterogeneous systems were studied in $[17,18]$. In [19-21], group consensus problems for different kinds of heterogeneous systems have been investigated, such as the discrete-time complex systems, the systems modeled 
by second-order, and Euler-Lagrange networks. In [22, 23], the authors studied the group consensus for the cooperative and heterogeneous networks with and without input time delays, respectively. In [24], group synchronization for linear and nonlinear heterogeneous systems was discussed via Lyapunov theory. In [25], second-order heterogeneous MASs in cooperation-competition network with switching topology are investigated. Adaptive and pinning control scheme were proposed as well. In [26], the authors also discussed the reverse group consensus for cooperation-competition network.

Inspired by the relevant research works, this paper will discuss the group consensus issues for the heterogeneous MASs with time delays. The following two cases are included: with communication and input time delays. As known, time delays inevitably exist in the complex systems. Meanwhile, they usually affect and even destroy the stability of complex systems [14-16]. Therefore, it is very important and necessary to discuss the group consensus problems with the influence of time delays. Our contributions are mainly listed as below: firstly, two novel delayed group consensus protocols are proposed, which is modeled by the agents' competitive relationship. The innovation is mainly embodied in the following two aspects: on the one hand, the related works [10-24] are based on the cooperative complex systems. On the other hand, there is a special case in the works [15, 21-23]; that is, the dynamics of the first-order agents are added with a virtual velocity estimation in order to make theoretical analysis more convenient. As a fact of that, this situation is not quite true. Hence, we relax it in our protocol. Secondly, we also relax the following two preconditions in [15, 20-24]: in-degree balance and the geometric multiplicity of zero eigenvalues of the Laplacian matrix associated with the adjacency matrix are at least two. They limit the topology of the system and the information interaction between the agents. As it does not rely on the restrict conditions, the case we discuss is more general. Lastly, by the matrix theory and frequency domain method, we theoretically propose some sufficient algebraic criteria for the achievement of couple-group consensus of the proposed complex systems. In general, our results should be more general.

The remaining parts of the paper are organised as follows. In Section 2, definition, lemma, and problem statements are listed firstly. In Section 3, we mainly discuss the couple-group consensus for the heterogeneous MASs with time delays. Subsequently, several simulated examples are illustrated to verify the correctness of our findings, and the conclusions are drawn finally in Section 5.

Notation. In the paper, let $\mathbb{R}, \mathbb{C}$ denote the real and complex numbers sets. $\forall z \in \mathbb{C}$, its modulus and the real part are represented by $|z|$ and $\operatorname{Re}(z) . I_{n}$ denotes $n$-dimensional identity matrix. $\lambda_{i}(\Lambda), \operatorname{det}(\Lambda)$ denote the $i$ th eigenvalue and the determinant of the matrix $\Lambda$, respectively.

\section{Problems Statement}

Consider a heterogeneous MASs containing $n+m$ agents; we conveniently assume the first $n$ and remaining $m$ agents are second-order and first-order agents, and their dynamics are presented as follows:

$$
\begin{aligned}
& \dot{\zeta}_{i}(t)=\varsigma_{i}(t), \\
& \dot{\zeta}_{i}(t)=u_{i}(t), \quad i \in \sigma_{1}, \\
& \dot{\zeta}_{i}(t)=u_{i}(t), \quad i \in \sigma_{2},
\end{aligned}
$$

where $\sigma_{1}=\{1,2, \ldots, n\}, \sigma_{2}=\{n+1, n+2, \ldots, n+m\}, \sigma=$ $\sigma_{1} \cup \sigma_{2} \cdot u_{i}(t), \zeta_{i}(t), \varsigma_{i}(t) \in \mathbb{R}$ are control input, position, and velocity state of the agent $i$, respectively.

In heterogeneous MASs, the neighbors of the agent $i$ may include second- and first-order agents, which can be described as $N_{i, s}$ and $N_{i, f}$, respectively. Hence, its neighbor set $N_{i}=N_{i, f} \cup N_{i, s}$. The adjacency matrix $A$ can be partitioned as $A=\left[\begin{array}{cc}A_{s} & A_{s f} \\ A_{f s} & A_{f}\end{array}\right]$, where $A_{s} \in \mathbb{R}^{n \times n}, A_{f} \in$ $\mathbb{R}^{m \times m}, A_{s f}$ represents the adjacency weights from the secondorder agents to the first-order agents, and $A_{f s}$ denotes the adjacency weights from the first-order agents to the second-order agents, respectively. The Laplacian matrix $L$ can also be rewritten as $L=D-A=\left[\begin{array}{cc}L_{s}+D_{s f} & -A_{s f} \\ -A_{f s} & L_{f}+D_{f s}\end{array}\right]$, where $L_{s}$ and $L_{f}$ denote the corresponding second-order and first-order agents' Laplacian matrix, respectively. $D_{s f}=$ $\operatorname{diag}\left\{\sum_{j \in N_{i, f}} a_{i j}, i \in \sigma_{1}\right\}, D_{f s}=\operatorname{diag}\left\{\sum_{j \in N_{i, s}} a_{i j}, i \in \sigma_{2}\right\}$. Therefore, when the topology of the system is a bipartite graph, the matrix $D+A$ associated with the system can be rewritten as $D+A=\left[\begin{array}{cc}L_{s}+D_{s f} & A_{s f} \\ A_{f s} & L_{f}+D_{f s}\end{array}\right]$.

Next, the definition and lemma will be listed to further our analysis.

Definition 1. Couple-group consensus for the heterogeneous MASs (1) is said to be reached asymptotically, if and only if the following two conditions are satisfied for any initial states:

$$
\begin{aligned}
& \lim _{t \rightarrow+\infty}\left\|\zeta_{i}(t)-\zeta_{j}(t)\right\|=0, \quad \text { if } i, j \in \sigma_{k}, k=1,2 ; \\
& \lim _{t \rightarrow+\infty}\left\|\zeta_{i}(t)-\zeta_{j}(t)\right\|=0, \quad \text { if } i, j \in \sigma_{k}, k=1,2 .
\end{aligned}
$$

Lemma 2 (see [9]). Consider a complex networks with $N$ agents, if its topology is a bipartite digraph and contains a directed spanning tree; it has $\operatorname{Re}\left(\lambda_{i}(D+A)\right)>0$, when $\lambda_{i}(D+$ $A) \neq 0$ and $\operatorname{rank}(D+A)=N-1$. In particular, if the topology is an undirected bipartite graph, the nonzero eigenvalues of the matrix $D+A$ are positive real numbers.

\section{Main Results}

In this section, we will discuss the couple-group consensus for the heterogeneous multiagent systems with communication and input time delays, respectively.

First, we will discuss the case where with communication time delays.

As is known the relationship between the agents in complex systems includes cooperative relationship, competitive relationship, and both of them. In the related works [1024 ], the cooperative and competitive relationship between the 
agents $i$ and $j$ is described as $x_{j}-x_{i}$ and $x_{j}+x_{i}$, respectively. In $[25,26]$, they also are presented by the coupling weight $a_{i j}$ between these two agents. If $a_{i j}>0$, it is shown that the relationship between the agent $i$ and $j$ is cooperative. If $a_{i j}<0$, it means competition. Most of the works focused on the systems constructed by cooperative relationship between the agents and there are few reports about competitive networks. Hence, motivated by the related works and on the foundation of systems (1), a novel group protocol for a class of heterogeneous MASs based on the competition relationship between the agents is proposed as follows:

$$
\begin{aligned}
& \dot{\zeta}_{i}(t)=\varsigma_{i}(t), \\
& \dot{\zeta}_{i}(t)=-\alpha\left[\sum_{j \in N_{i}} a_{i j}\left[\zeta_{j}\left(t-\tau_{i j}\right)+\zeta_{i}(t)\right]\right]-\beta \varsigma_{i}(t), \\
& \quad i \in \sigma_{1}, \\
& \dot{\zeta}_{i}(t)=-\gamma\left[\sum_{j \in N_{i}} a_{i j}\left[\zeta_{j}\left(t-\tau_{i j}\right)+\zeta_{i}(t)\right]\right], \quad i \in \sigma_{2},
\end{aligned}
$$

where $\tau_{i j}$ denotes the communication time delay between the agents $i$ and $j . \alpha, \beta, \gamma>0$ are the control parameters of the systems.

Theorem 3. If $\max \left\{d_{i}\right\}<\beta^{2} / 2 \alpha, i \in \sigma_{1}$, and $\gamma>0$ hold, the couple-group consensus for systems (3) with an undirected bipartite topology can be achieved asymptotically.

Proof. Taking the Laplace transforms to (3), it yields that

$$
\begin{aligned}
& s \zeta_{i}(s)=\zeta_{i}(s), \\
& s \zeta_{i}(s)=-\alpha\left[\sum_{j \in N_{i}} a_{i j}\left[e^{-\tau_{i j} s} \zeta_{j}(s)+\zeta_{i}(s)\right]\right]-\beta \zeta_{i}(s), i \in \sigma_{1}, \\
& s \zeta_{i}(s)=-\gamma\left[\sum_{j \in N_{i}} a_{i j}\left[e^{-\tau_{i j} s} \zeta_{j}(s)+\zeta_{i}(s)\right]\right], \quad i \in \sigma_{2},
\end{aligned}
$$

where $\zeta_{i}(s), \varsigma_{i}(s)$ represent the Laplace transform of $\zeta_{i}(t), \varsigma_{i}(t)$, respectively.

Define

$$
\begin{aligned}
\zeta_{s}(s) & =\left[\zeta_{1}(s), \zeta_{2}(s), \ldots, \zeta_{n}(s)\right]^{T}, \\
\zeta_{f}(s) & =\left[\zeta_{n+1}(s), \zeta_{n+2}(s), \ldots, \zeta_{n+m}(s)\right]^{T}, \\
\widehat{L} & =\left(\widehat{l}_{i j}\right)_{(n+m) \times(n+m)}= \begin{cases}e^{-\tau_{i j} s} a_{i j}, & i \neq j, \\
\sum_{j \in N_{i}} a_{i j}, & i=j,\end{cases} \\
y(s) & =\left[\zeta_{s}^{T}(s), \zeta_{f}^{T}(s)\right]^{T} ;
\end{aligned}
$$

then we have

$$
s y(s)=\widetilde{\Phi}(s) y(s),
$$

where

$$
\widetilde{\Phi}(s)=\left[\begin{array}{cc}
\frac{-s^{2} I_{n}-\alpha\left(\widehat{L}_{s}+\widehat{D}_{s f}\right)}{\beta} & \frac{-\alpha \widehat{A}_{s f}}{\beta} \\
-\gamma \widehat{A}_{f s} & -\gamma\left(\widehat{L}_{f}+\widehat{D}_{f s}\right)
\end{array}\right] .
$$

Define $\widetilde{F}(s)=\operatorname{det}(s I-\widetilde{\Phi}(s))$. It is known that the systems' couple-group consensus will be achieved if the roots of $\widetilde{F}(s)$ either are at $s=0$ or have negative real parts by the stability theory.

When $s=0, \widetilde{F}(0)=(-\alpha / \beta)^{n}(-\gamma)^{m} \operatorname{det}(D+A)$. Based on Lemma 2, we know that the roots of $\widetilde{F}(0)$ are at $s=0$ if the topology of the systems is an undirected bipartite graph.

When $s \neq 0$, define $\widetilde{F}(s)=\operatorname{det}(I+H(s))$, where $H(s)=$ $-\widetilde{\Phi}(s) / s$. Then it follows that

$$
H(s)=\left[\begin{array}{cc}
\frac{s^{2} I_{n}+\alpha\left(\widehat{L}_{s}+\widehat{D}_{s f}\right)}{s \beta} & \frac{\alpha \widehat{A}_{s f}}{s \beta} \\
\frac{\gamma \widehat{A}_{f s}}{s} & \frac{\gamma\left(\widehat{L}_{f}+\widehat{D}_{f s}\right)}{s}
\end{array}\right] .
$$

Set $s=j \omega$, based on the general Nyquist criterion, it is known that if and only if the point $(-1, j 0)$ is not enclosed by the Nyquist curve of $H(j \omega)$, the roots of $\widetilde{F}(s)$ are located in the open left-half plane. Namely, the roots of $\widetilde{F}(s)$ have negative real parts. Hence, according to the Gershgorin Disk Theorem, we have

$$
\lambda(H(j \omega)) \in\left\{H_{i}, i \in \sigma_{1}\right\} \cup\left\{H_{i}, i \in \sigma_{2}\right\} .
$$

When $i \in \sigma_{1}$, it yields that

$$
\begin{aligned}
H_{i} & =\left\{\zeta: \zeta \in\left|\zeta-\frac{\alpha}{j \omega \beta} \sum_{j \in N_{i}} a_{i j}-\frac{j \omega}{\beta}\right|\right. \\
& \left.\leq \sum_{j \in N_{i}}\left|\frac{\alpha a_{i j}}{j \omega \beta} e^{-j \omega \tau_{i j}}\right|\right\} .
\end{aligned}
$$

Define $\sum_{j \in N_{i}} a_{i j}=d_{i}, i \in \sigma_{1}$; since the point $(-a, j 0)$, $a \geq 1$ is not enclosed in $H_{i}, i \in \sigma_{1}$, the following inequation (11) can be obtained:

$$
\left|-a-\frac{\alpha d_{i}}{j \omega \beta}-\frac{j \omega}{\beta}\right|>\sum_{j \in N_{i}}\left|\frac{\alpha a_{i j}}{j \omega \beta} e^{-j \omega \tau_{i j}}\right| .
$$

From (11), it follows that

$$
\left|-a+\frac{\alpha d_{i}}{\omega \beta} j-\frac{j \omega}{\beta}\right|>\left|\frac{\alpha d_{i}}{j \omega \beta}\left(\cos \omega \tau_{i j}-j \sin \omega \tau_{i j}\right)\right| .
$$

After some manipulation, it yields that

$$
a^{2}+\frac{\omega^{2}}{\beta^{2}}-\frac{2 \alpha d_{i}}{\beta^{2}}>0 \text {. }
$$


As $a \geq 1$, hence, we have

$$
1+\frac{\omega^{2}}{\beta^{2}}-\frac{2 \alpha d_{i}}{\beta^{2}}>0
$$

As $\beta>0$, the following inequations can be obtained:

$$
\beta^{2}-2 \alpha d_{i}>0
$$

From (15), the condition $d_{i}<\beta^{2} / 2 \alpha$ can be obtained. When $i \in \sigma_{2}$, it yields that

$$
H_{i}=\left\{\zeta: \zeta \in \| \zeta-\frac{\gamma}{j \omega} \sum_{j \in N_{i}} a_{i j}\left|\leq \sum_{j \in N_{i}}\right| \frac{\gamma a_{i j}}{j \omega} e^{-j \omega \tau_{i j}} \mid\right\} .
$$

Define $\sum_{j \in N_{i}} a_{i j}=\widetilde{d}_{i}, i \in \sigma_{2}$; if the point $(-a, j 0), a \geq 1$ is not enclosed in $H_{i}, i \in \sigma_{2}$, we have

$$
a^{2}>0
$$

It is easy to know that inequation (17) can be satisfied for $a \geq 1$.

From what have been discussed above, that completes the proof of Theorem 3 .

Corollary 4. For systems (3) with a directed bipartite topology which contains a directed spanning tree, if $\max \left\{d_{i}\right\}<\beta^{2} / 2 \alpha$, $i \in \sigma_{1}$, and $\gamma>0$ hold, the couple-group consensus of the systems can be achieved asymptotically.

We omit the proof progress here due to the space limitation.

Remark 5. From the results in Theorem 3 and Corollary 4, we can find that the achievement of the couple-group consensus relies on the control parameters and the second-order agents' in-degree. Meanwhile, communication time delays cannot affect the achievement of the group consensus.

Next, we will investigate the couple-group consensus problem for the heterogeneous MASs with input time delays.

Based on systems (3), a delayed protocol which based on the competitive relationship between the agents is designed as follows:

$$
\begin{aligned}
& \dot{\zeta}_{i}(t)=\zeta_{i}(t), \\
& \dot{\zeta}_{i}(t)=-\alpha\left[\sum_{j \in N_{i}} a_{i j}\left[\zeta_{j}(t)+\zeta_{i}(t-\tau)\right]\right]-\beta \zeta_{i}(t-\tau), \\
& \dot{\zeta}_{i}(t)=-\gamma\left[\sum _ { j \in N _ { i } } a _ { i j } \left[\zeta_{j}(t)+\zeta_{1},\right.\right.
\end{aligned}
$$

where $\tau$ denotes the input time delays of the agent i. $\alpha, \beta, \gamma>$ 0 are the control parameters of the systems. Note that we just discuss the special case.
Theorem 6. If $\max \left\{d_{i}\right\}<\beta^{2} / 2 \alpha$ and $\tau \in[0, \min \{1 / 2 \beta$, $\left.\left.\max \left\{1 / 2 \gamma \widetilde{d}_{i}\right\}\right\}\right]$ can be held, couple-group consensus for the heterogeneous MASs (18) with an undirected bipartite graph topology can be achieved asymptotically, where $d_{i}=\sum_{j \in N_{i}} a_{i j}$, $i \in \sigma_{1}$, and $\widetilde{d}_{i}=\sum_{j \in N_{i}} a_{i j}, i \in \sigma_{2}$.

Proof. Taking the Laplace transforms to (18), it yields that

$$
\begin{aligned}
& s \zeta_{i}(s)= \varsigma_{i}(s), \\
& s \zeta_{i}(s)=-\alpha\left[\sum_{j \in N_{i}} a_{i j}\left[\zeta_{j}(s)+e^{-\tau s} \zeta_{i}(s)\right]\right]-\beta e^{-\tau s} \varsigma_{i}(s), \quad i \in \sigma_{1}, \\
& s \zeta_{i}(s)=-\gamma\left[\sum_{j \in N_{i}} a_{i j}\left[\zeta_{j}(s)+e^{-\tau s} \zeta_{i}(s)\right]\right], \quad i \in \sigma_{2},
\end{aligned}
$$

where $\zeta_{i}(s), \varsigma_{i}(s)$ represent the Laplace transform of $\zeta_{i}(t), \varsigma_{i}(t)$, respectively.

As the proof progress is similar with that in Theorem 3, we omit it here due to the space limitation.

Corollary 7. If the conditions $\max \left\{d_{i}\right\}<\beta^{2} / 2 \alpha$ and $\tau \in$ $\left[0, \min \left\{1 / 2 \beta, \max \left\{1 / 2 \gamma \widetilde{d}_{i}\right\}\right\}\right]$ are satisfied, couple-group consensus for the heterogeneous MASs (18) with a bipartite digraph topology which has a directed spanning tree can be achieved asymptotically, where $d_{i}=\sum_{j \in N_{i}} a_{i j}, i \in \sigma_{1}$, and $\widetilde{d}_{i}=\sum_{j \in N_{i}} a_{i j}$, $i \in \sigma_{2}$.

Remark 8. From the proof progress of theorems and corollaries, it is found that we absolutely relax the following two restrictive assumptions which existed in [15, 20-24]: in-degree balance and the geometric multiplicity of zero eigenvalues of the systems Laplacian matrix are at least two. It is known that in-degree balance means that there is no actual communication between the clusters. Meanwhile, the later condition also restricts the topology of the systems. As both of them are very restrictive conditions, our results will be more general.

Remark 9. From the results in Theorem 6 and Corollary 7 , we know that the achievement of couple-group consensus relies on control parameters of the systems, in-degree of the second-order agents, and the input time delay. Meanwhile, the upper bound of the input time delay is determined by the control parameters and the in-degree of the first-order agents.

Remark 10. It is worth noting that there is a virtual velocity estimation in the dynamics of the first-order agents for the convenience of analysis in [15, 21-23]. In fact, this situation is so ideal that it cannot meet the requirement in applications. In our models, we relax it. Therefore, the protocols we proposed are more practical. Meanwhile, the work will become more challenging when some preconditions are removed. 


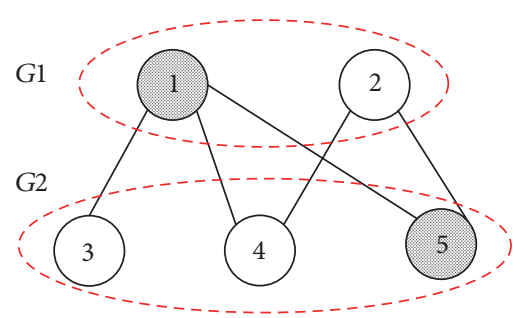

FIgURE 1: The bipartite topology of the heterogeneous MASs.

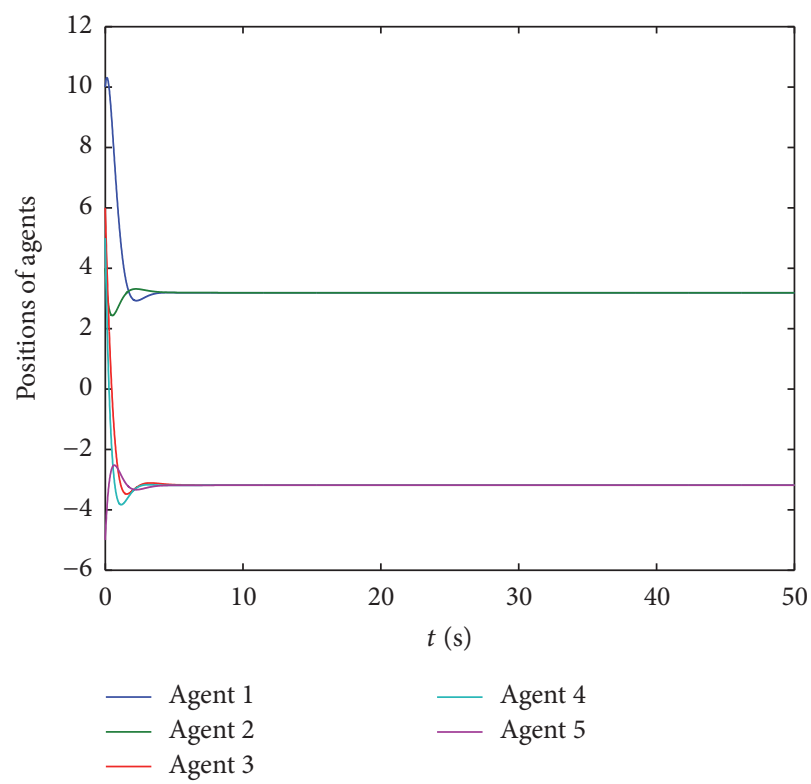

(a)

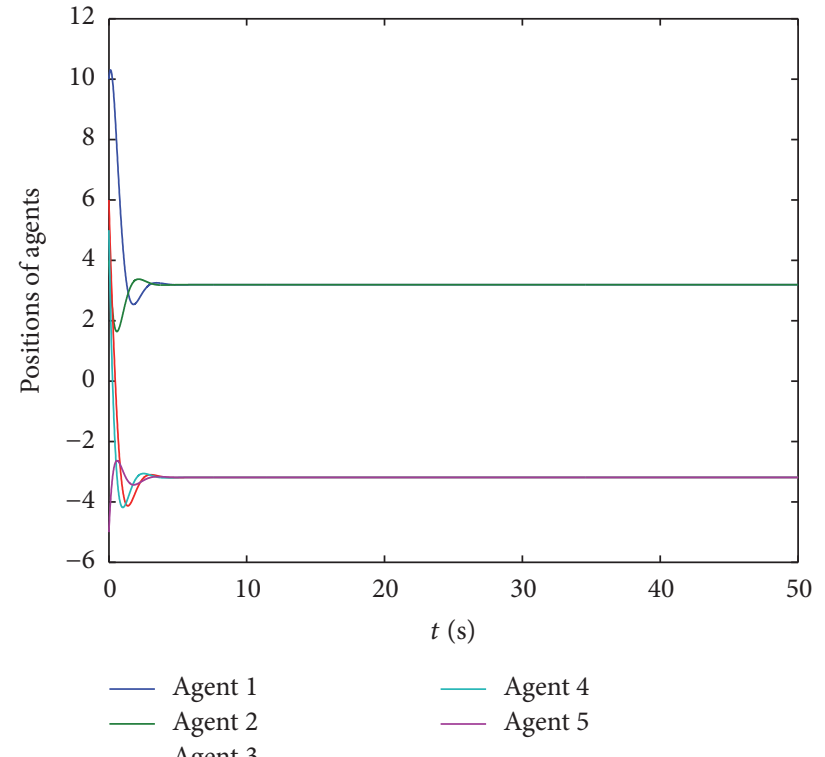

(b)

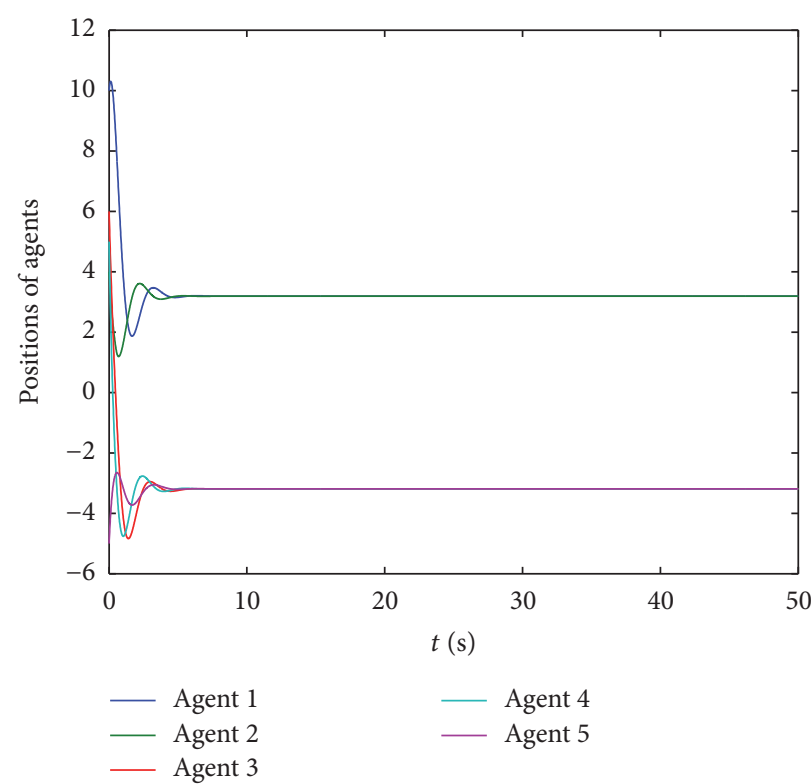

(c)

FIgURE 2: The state trajectories of the agents in systems (3). (a) $\tau_{i j}=0 \mathrm{~s}$, (b) $\tau_{i j}=0.3 \mathrm{~s}$, and (c) $\tau_{i j}=0.5 \mathrm{~s}$. 


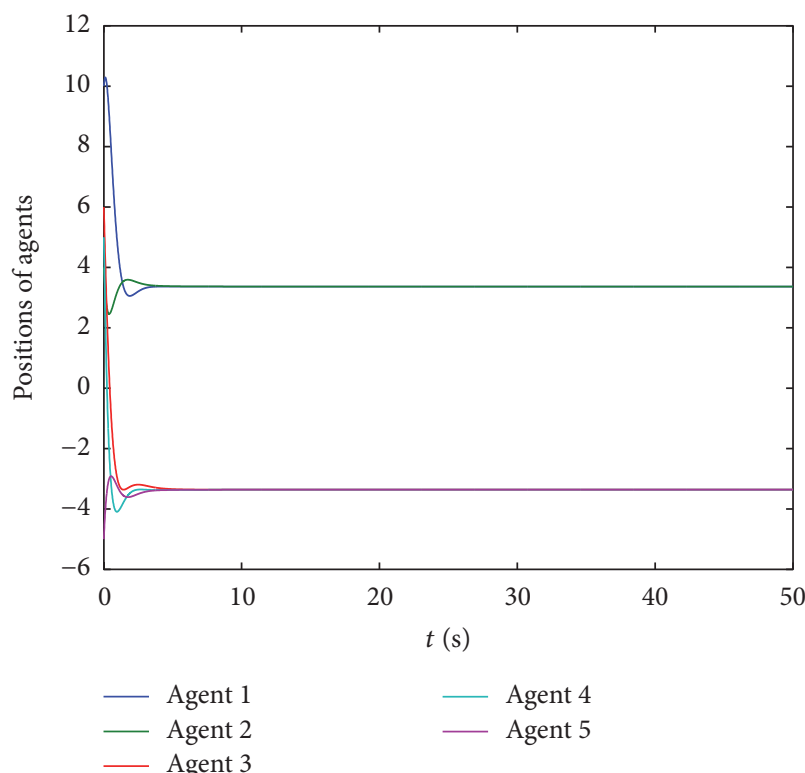

(a)

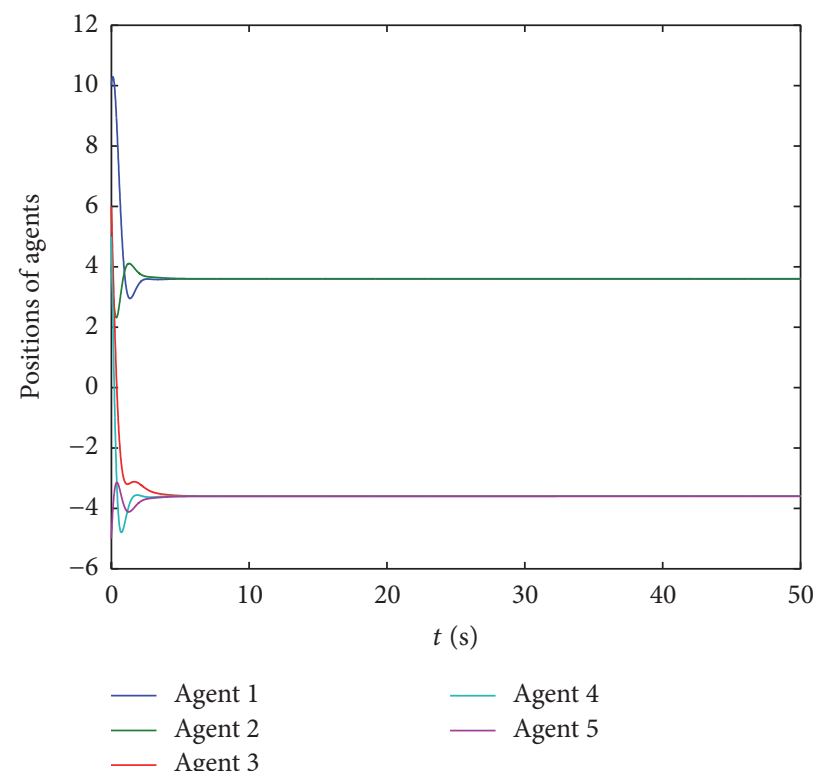

(b)

Figure 3: The state trajectories of the agents in systems (18). (a) $\tau=0.1 \mathrm{~s}$ and (b) $\tau=0.2 \mathrm{~s}$.

\section{Simulation Results}

In the section, we will illustrate the correctness of the obtained results by several simulated examples.

Suppose the heterogeneous systems with the undirected bipartite topology shown in Figure 1. For convenience, we also assume agents 1 and 5 are second-order and agents 2, 3, and 4 are first-order. In this situation, clusters $G 1$ and $G 2$ are heterogeneous as well.

Set $\alpha=1, \beta=2.5, \gamma=1$, and $a_{i j}=1, i, j \in[1,5]$. From Figure 1 , we have $d_{1}=3, d_{2}=2, d_{3}=1, d_{4}=2$, and $d_{5}=2$.

Example 1. It is easy to verify that the conditions in Theorem 3 are satisfied. As the communication time delays cannot affect the achievement of the systems, in the example, we suppose the communication time delays between the agents are equal. Meanwhile, in order to describe the effect of the communication time delay on systems' convergence rate, we mainly consider the following three cases: (1) $\tau_{i j}=0 \mathrm{~s}$; (2) $\tau_{i j}=0.3 \mathrm{~s}$; (3) $\tau_{i j}=0.5 \mathrm{~s}$. The trajectories of the agents in systems (3) are shown in Figure 2, respectively. The results show that the systems' couple-group consensus is reached, respectively. Moreover, we can find that the systems' convergence rate will be improved with the decrease of communication delay.

Example 2. From Theorem 6, we know $\tau \in[0,1 / 5]$. Hence, in the simulation, we choose $\tau=0.1 \mathrm{~s}$ and $\tau=0.2 \mathrm{~s}$, respectively. It is known that all the conditions in Theorem 6 can be satisfied. The trajectories of the agents in systems (18) are shown in Figure 3, respectively. The results show that the systems' couple-group consensus is reached, respectively. Furthermore, with the increase of input time delay, the convergence rate of the systems will decrease accordingly.

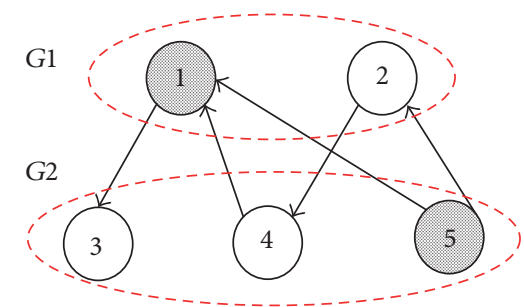

FIGURE 4: The directed bipartite topology of the heterogeneous MASs.

Example 3. Consider systems (3) with the topology shown in Figure 4. It is a bipartite digraph and contains a directed spanning tree. In this example, all the parameters are set as those in Example 1. It is easy to verify that the conditions in Corollary 4 are satisfied as well. The trajectories of the agents in systems (3) with different communication time delays are shown in Figure 5, respectively. From the results illustrated in Figure 5, we can find the couple-group consensus can be reached asymptotically, respectively.

Example 4. Based on Example 2, we suppose systems (18) with the topology shown in Figure 4 and set $\tau=0.1 \mathrm{~s}$ and $\tau=0.2 \mathrm{~s}$, respectively. It is known that all the conditions in Corollary 7 can be satisfied. The trajectories of the agents in systems (18) are shown in Figure 6, respectively. The results show that the systems' couple-group consensus is reached, respectively.

Example 5. In this example, we will discuss the other case where systems (18) with the topology are shown in Figure 7. It is shown that the second-order agents 1 and 2 belong to cluster $G 1$. Similarly, cluster $G 2$ contains the first-order agents 


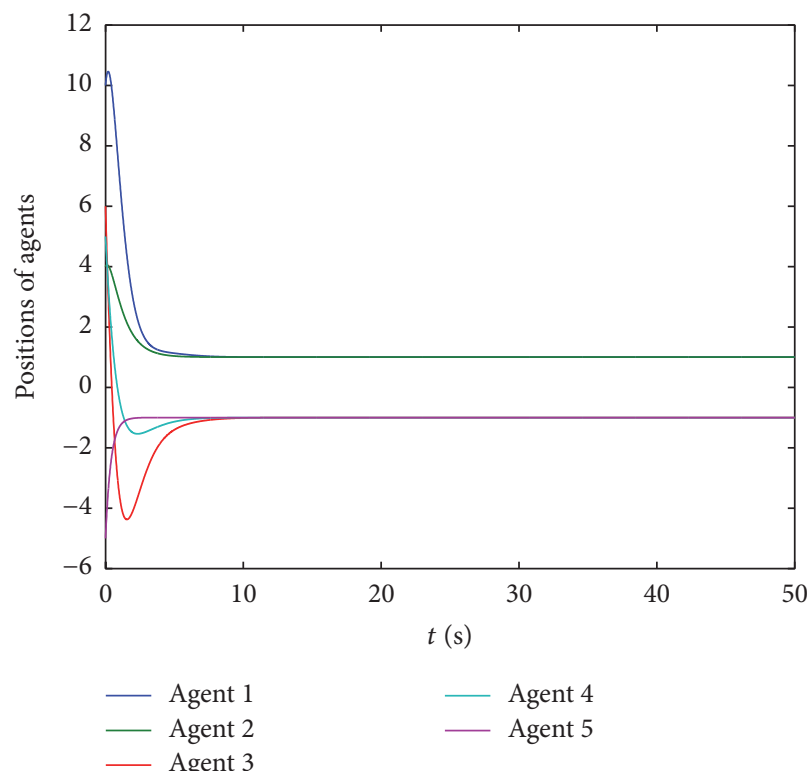

(a)

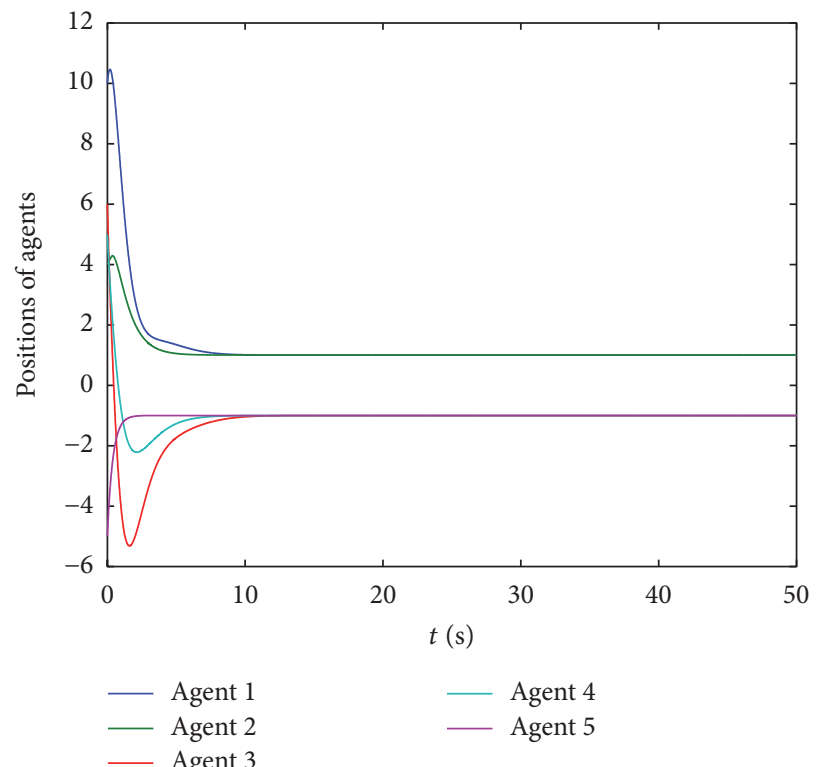

(b)

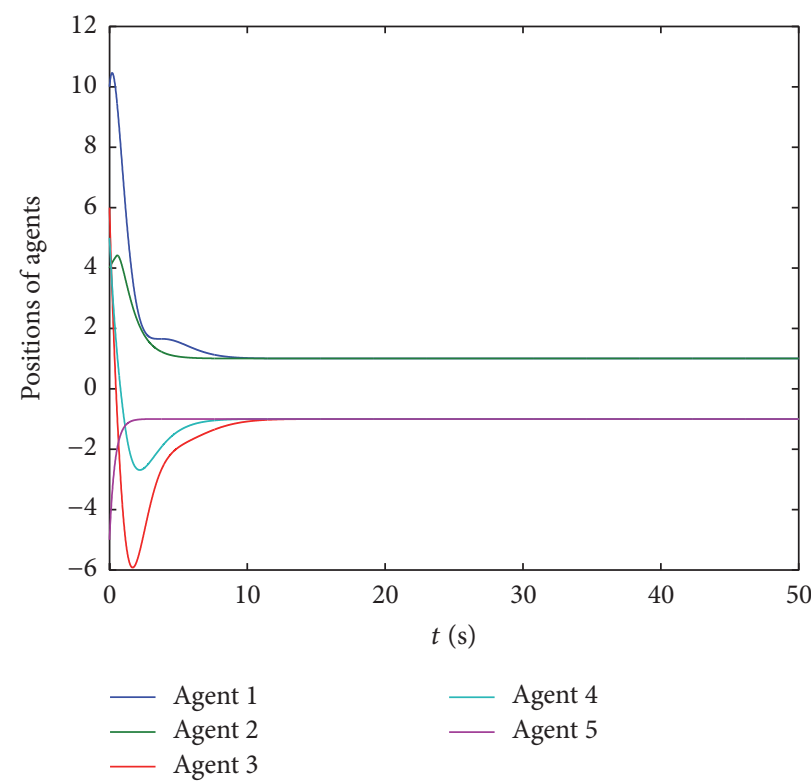

(c)

Figure 5: The state trajectories of the agents in systems (3). (a) $\tau_{i j}=0 \mathrm{~s}$, (b) $\tau_{i j}=0.3 \mathrm{~s}$, and (c) $\tau_{i j}=0.5 \mathrm{~s}$.

3, 4 and 5. Namely, the whole system is heterogeneous, but its two clusters are homogeneous. Based on Examples 2 and 4, we also set $\tau=0.1 \mathrm{~s}$ and $\tau=0.2 \mathrm{~s}$, respectively. Meanwhile, the other parameters coincide with those in Example 2. It is not difficult to find that the conditions in Corollary 7 are satisfied. The trajectories of the agents in systems (18) are illustrated in Figures 8 and 9, respectively. From the results, they show that the couple-group consensus of the systems can be achieved asymptotically.

Remark 11. We assume each subgroup is heterogeneous in Examples 1-4. Note that our results also apply to the case where the clusters are homogeneous. At this point, the position and velocity states of the agents will achieve group consensus in the cluster containing second-order agents. The results in Example 5 can be explained here.

\section{Conclusions}

In this paper, we investigate the couple-group consensus problems for the heterogeneous MASs with communication and input time delays, respectively. Based on the competitive relationship between the agents, two novel delayed group consensus protocols are designed. Specifically, some sufficient algebraic criteria are obtained by the matrix theory and frequency domain method in the case of relaxing the 


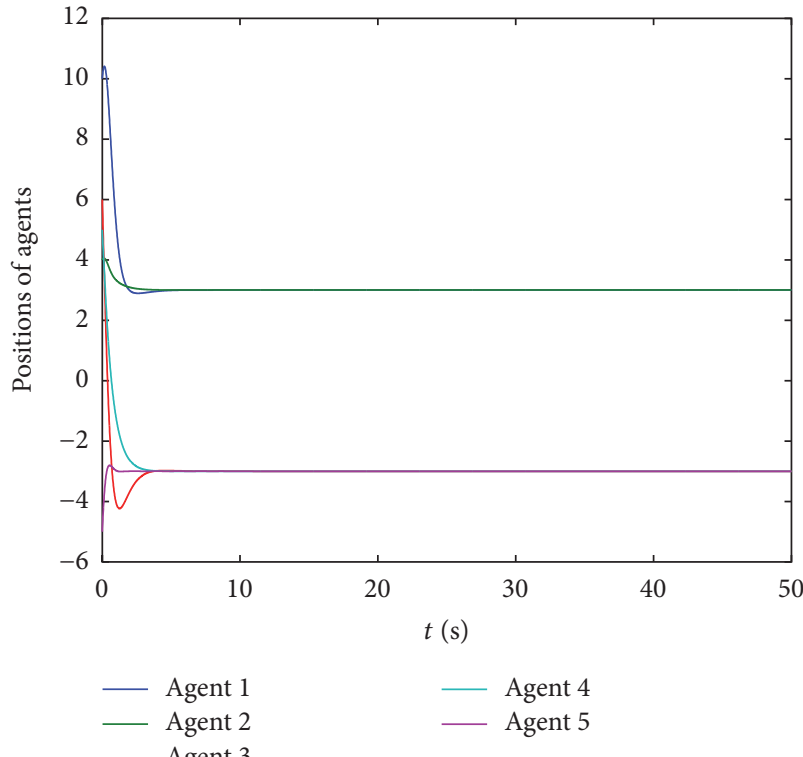

(a)

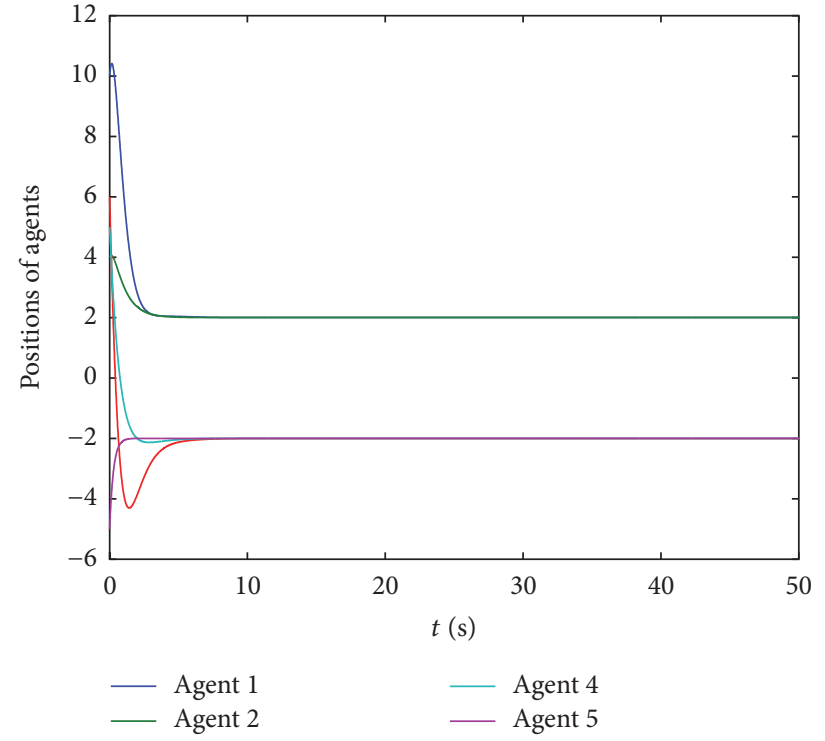

(b)

Figure 6: The state trajectories of the agents in systems (18). (a) $\tau=0.1 \mathrm{~s}$ and (b) $\tau=0.2 \mathrm{~s}$.

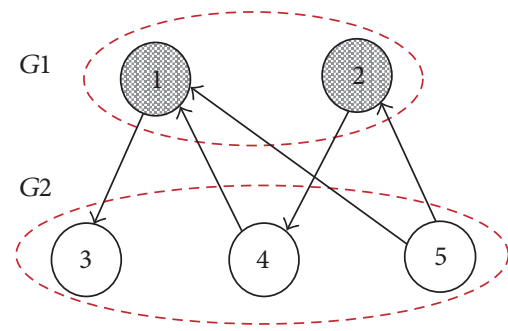

FIGURE 7: The directed bipartite topology of the heterogeneous MASs.

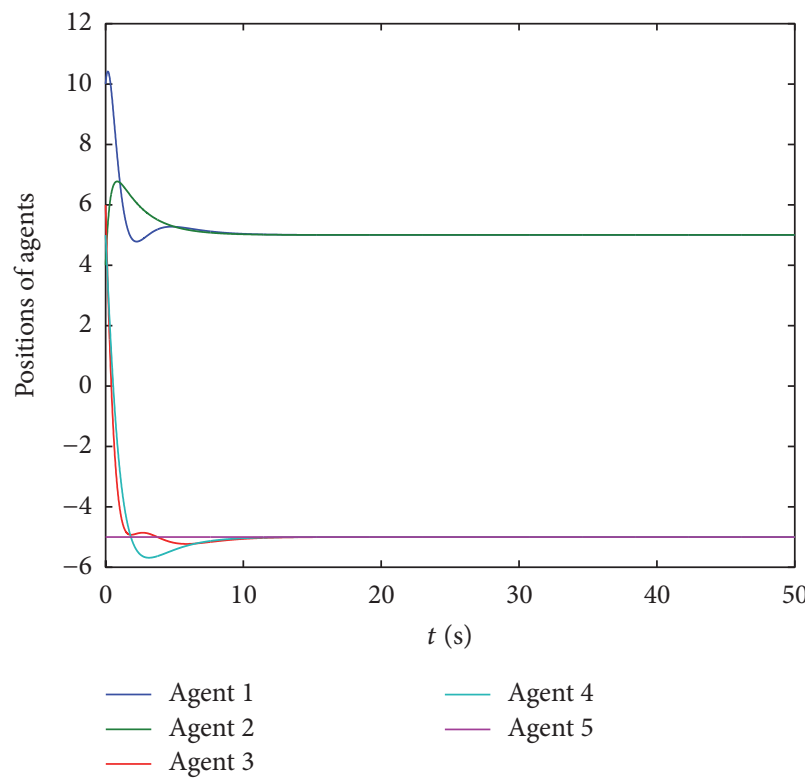

(a)

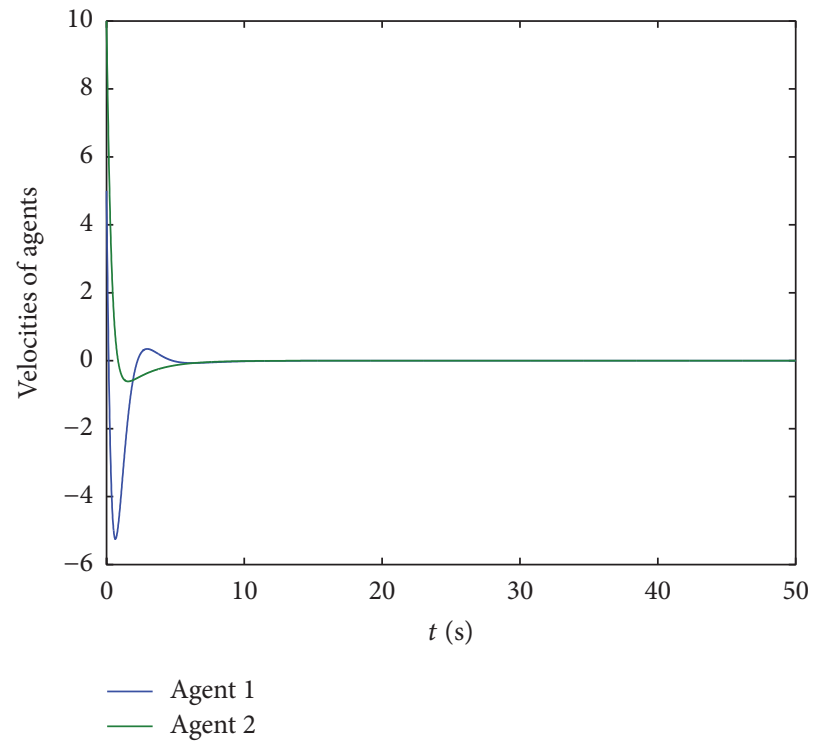

(b)

FIgURE 8: The state trajectories of the agents in systems (18) when $\tau=0.1 \mathrm{~s}$. (a) Position state and (b) velocity state. 


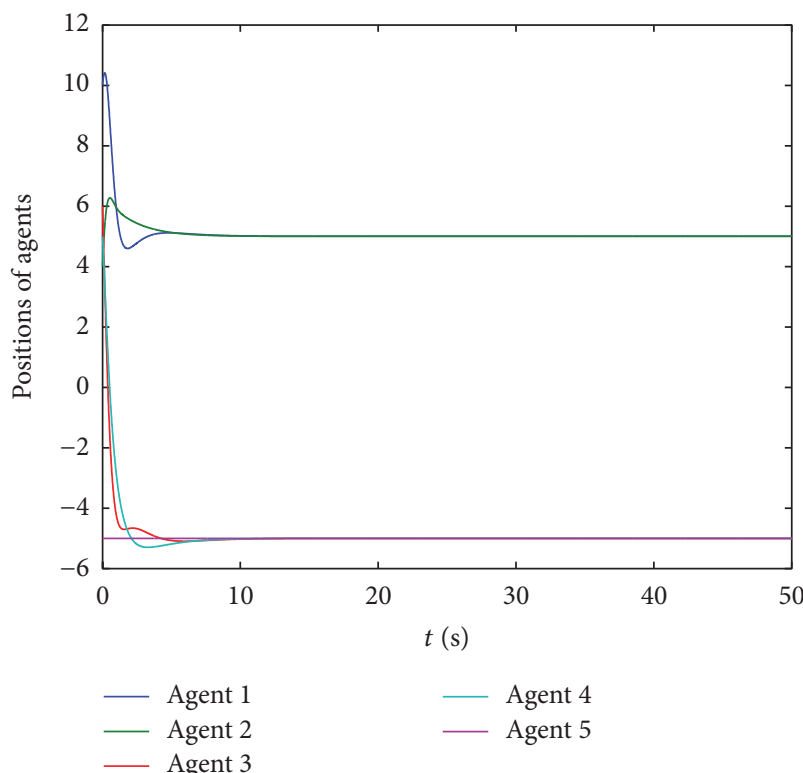

(a)

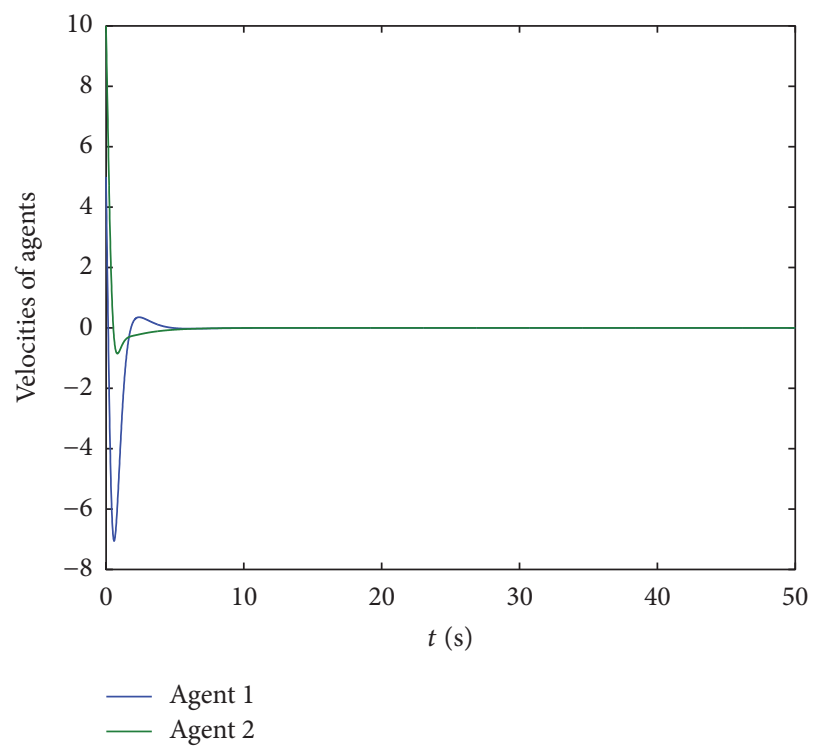

(b)

FIGURE 9: The state trajectories of the agents in systems (18) when $\tau=0.2 \mathrm{~s}$. (a) Position state and (b) velocity state.

restrictive preconditions existing in the related works. From the results, we find that the achievement of the couple-group consensus depends on the second-order agents in-degree and the control parameters of the systems, whereas it is independent of the communication time delays. Meanwhile, the upper bound of the input time delay is determined by the control parameters of the systems and the in-degree of the first-order agents. With the increase of the in-degree of the first-order agents, the tolerance of the systems to the input time delay will be reduced. It reveals that we should improve the data processing capability of the key agents. Furthermore, through the simulated examples, it is found that the smaller the time delays are including input and communication time delays, the faster the systems will converge. In the future, we will further our work to the cases with multiple time delays, switching topology, and so on.

\section{Conflicts of Interest}

The authors declare that they have no conflicts of interest.

\section{Acknowledgments}

This work was supported in part by the National Natural Science Foundation of China under Grants 61472464, 61572091, 61503308, and 61673080, in part by the Natural Science Foundation Project of Chongqing Science and Technology Commission under Grants nos. cstc2014jcyjA 40047 and cstc2015jcyjA40043, and in part by the Scientific and Technological Research Program of Chongqing Municipal Education Commission under Grant KJ1400429 and awarded in part by State Scholarship Fund of China Scholarship Council.

\section{References}

[1] W. Qiao and R. Sipahi, "Consensus control under communication delay in a three-robot system: design and experiments," IEEE Transactions on Control Systems Technology, vol. 24, no. 2, pp. 687-694, 2016.

[2] L. Jin and S. Li, "Distributed Task Allocation of Multiple Robots: A Control Perspective," IEEE Transactions on Systems, Man, and Cybernetics: Systems, pp. 1-9.

[3] C. Li, X. Yu, W. Yu, T. Huang, and Z.-W. Liu, "Distributed eventtriggered scheme for economic dispatch in smart grids," IEEE Transactions on Industrial Informatics, vol. 12, no. 5, pp. 17751785, 2016.

[4] G. Wen, Y. Zhao, Z. Duan, W. Yu, and G. Chen, "Containment of higher-order multi-leader multi-agent systems: a dynamic output approach," Institute of Electrical and Electronics Engineers Transactions on Automatic Control, vol. 61, no. 4, pp. 1135-1140, 2016.

[5] L. Ji, Q. Liu, and X. Liao, "On reaching group consensus for linearly coupled multi-agent networks," Information Sciences, vol. 287, pp. 1-12, 2014.

[6] X. Liao and L. Ji, "On pinning group consensus for dynamical multi-agent networks with general connected topology," Neurocomputing, vol. 135, no. 5, pp. 262-267, 2014.

[7] L. Ji, Y. Tang, Q. Liu, and X. Liao, "On adaptive pinning consensus for dynamic multi-agent networks with general connected topology," in Proceedings of the 2016 International Joint Conference on Neural Networks, IJCNN 2016, pp. 52815287, Canada, July 2016.

[8] L. Ji, Y. Tang, and Q. Liu, "On hybrid adaptive and pinning consensus for multiagent networks," Mathematical Problems in Engineering, vol. 2016, Article ID 9127356, 11 pages, 2016.

[9] Q. Wang and Y. Wang, "Cluster synchronization of a class of multi-agent systems with a bipartite graph topology," Science China Information Sciences, vol. 57, no. 1, pp. 1-11, 2014. 
[10] J. Lunze, "Synchronization of heterogeneous agents," Institute of Electrical and Electronics Engineers Transactions on Automatic Control, vol. 57, no. 11, pp. 2885-2890, 2012.

[11] L. Wang, M. Z. Q. Chen, and Q.-G. Wang, "Bounded synchronization of a heterogeneous complex switched network," Automatica, vol. 56, pp. 19-24, 2015.

[12] D. Goldin and J. Raisch, "Consensus for agents with double integrator dynamics in heterogeneous networks," Asian Journal of Control, vol. 16, no. 1, pp. 30-39, 2014.

[13] L. Wang, W.-J. Feng, M. Z. Chen, and Q.-G. Wang, "Global bounded consensus in heterogeneous multi-agent systems with directed communication graph," IET Control Theory \& Applications, vol. 9, no. 1, pp. 147-152, 2015.

[14] B. Cui, T. Ma, F. L. Lewis, C. Zhao, Y. Song, and C. Feng, "Distributed adaptive consensus control of heterogeneous multiagent chaotic systems with unknown time delays," IET Control Theory \& Applications, vol. 9, no. 16, pp. 2414-2422, 2015.

[15] C.-L. Liu and F. Liu, "Dynamical consensus seeking of heterogeneous multi-agent systems under input delays," International Journal of Communication Systems, vol. 26, no. 10, pp. 1243-1258, 2013.

[16] Y.-J. Sun, G.-L. Zhang, and J. Zeng, "Consensus analysis for a class of heterogeneous multiagent systems with time delay based on frequency domain method," Mathematical Problems in Engineering, vol. 2014, Article ID 248684, 2014.

[17] J. M. Kim, J. B. Park, and Y. H. Choi, "Leaderless and leaderfollowing consensus for heterogeneous multi-agent systems with random link failures," IET Control Theory \& Applications, vol. 8, no. 1, pp. 51-60, 2014.

[18] S. Li, G. Feng, X. Luo, and X. Guan, "Output consensus of heterogeneous linear discrete-time multiagent systems with structural uncertainties," IEEE Transactions on Cybernetics, vol. 45, no. 12, pp. 2868-2879, 2015.

[19] H.-X. Hu, W. Yu, Q. Xuan, C.-G. Zhang, and G. Xie, "Group consensus for heterogeneous multi-agent systems with parametric uncertainties," Neurocomputing, vol. 142, pp. 383-392, 2014.

[20] Y. Zheng and L. Wang, "A novel group consensus protocol for heterogeneous multi-agent systems," International Journal of Control, vol. 88, no. 11, pp. 2347-2353, 2015.

[21] C. Liu, Q. Zhou, and X. Hu, "Group consensus of heterogeneous multi-agent systems with fixed topologies," International Journal of Intelligent Computing and Cybernetics, vol. 8, no. 4, pp. 294-311, 2015.

[22] G. Wen, J. Huang, C. Wang, Z. Chen, and Z. Peng, "Group consensus control for heterogeneous multi-agent systems with fixed and switching topologies," International Journal of Control, vol. 89, no. 2, pp. 259-269, 2016.

[23] G. Wen, Y. Yu, Z. Peng, and H. Wang, "Dynamical group consensus of heterogenous multi-agent systems with input time delays," Neurocomputing, vol. 175, pp. 278-286, 2015.

[24] J. Qin, Q. Ma, H. Gao, Y. Shi, and Y. Kang, "On group synchronization for interacting clusters of heterogeneous systems," IEEE Transactions on Cybernetics, vol. 47, no. 12, 2016.

[25] H.-X. Hu, Q. Xuan, W. Yu, C.-G. Zhang, and G. Xie, "Secondorder consensus for heterogeneous multi-agent systems in the cooperation-competition network: a hybrid adaptive and pinning control approach," Nonlinear Analysis: Hybrid Systems, vol. 20, pp. 21-36, 2016.

[26] H.-X. Hu, W. Yu, G. Wen, Q. Xuan, and J. Cao, "Reverse group consensus of multi-agent systems in the cooperationcompetition network," IEEE Transactions on Circuits and Systems I: Regular Papers, vol. 63, no. 11, pp. 2036-2047, 2016. 


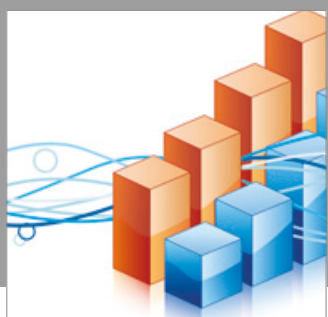

Advances in

Operations Research

vatersals

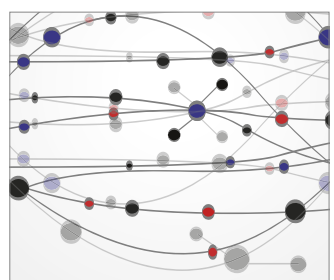

\section{The Scientific} World Journal
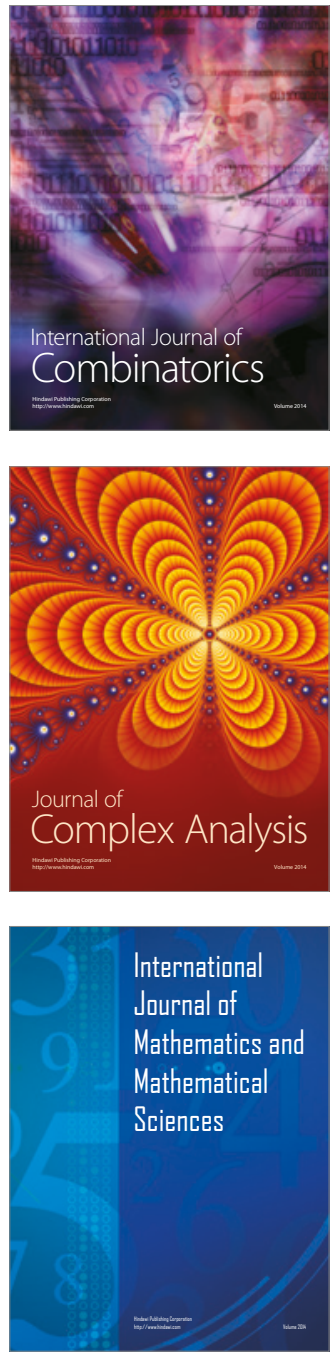
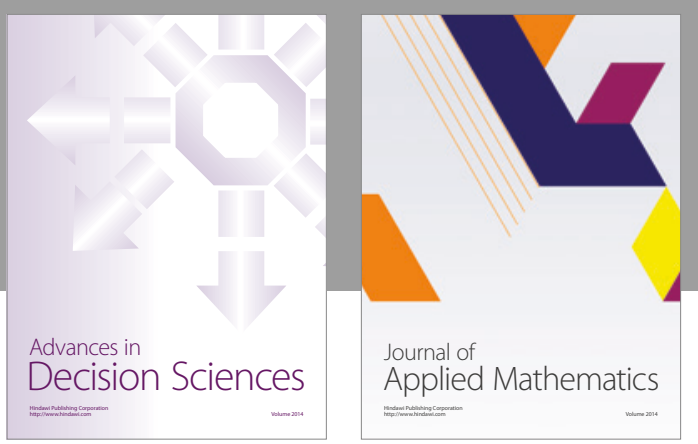

Algebra

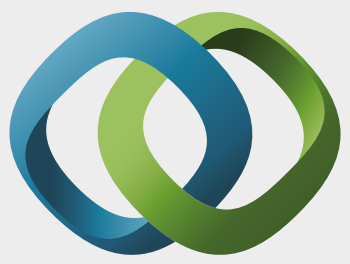

\section{Hindawi}

Submit your manuscripts at

https://www.hindawi.com
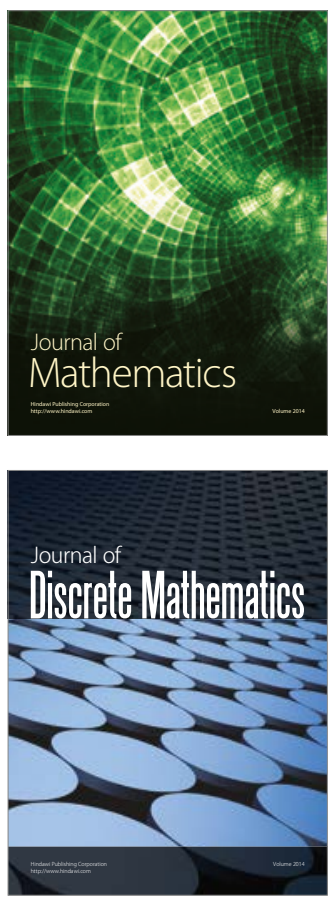

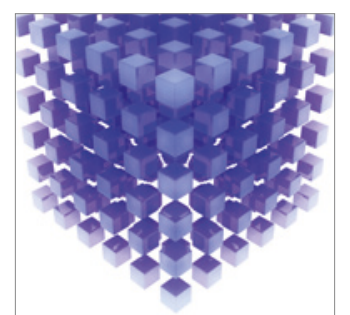

Mathematical Problems in Engineering
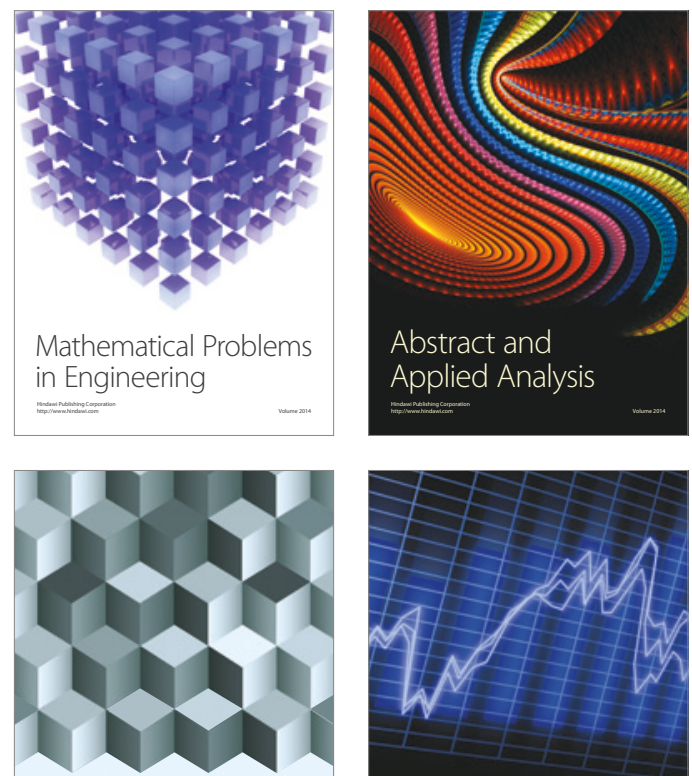

Journal of

Function Spaces

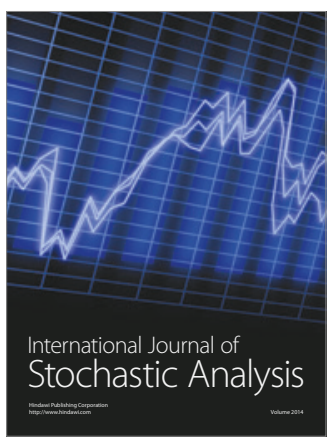

Probability and Statistics
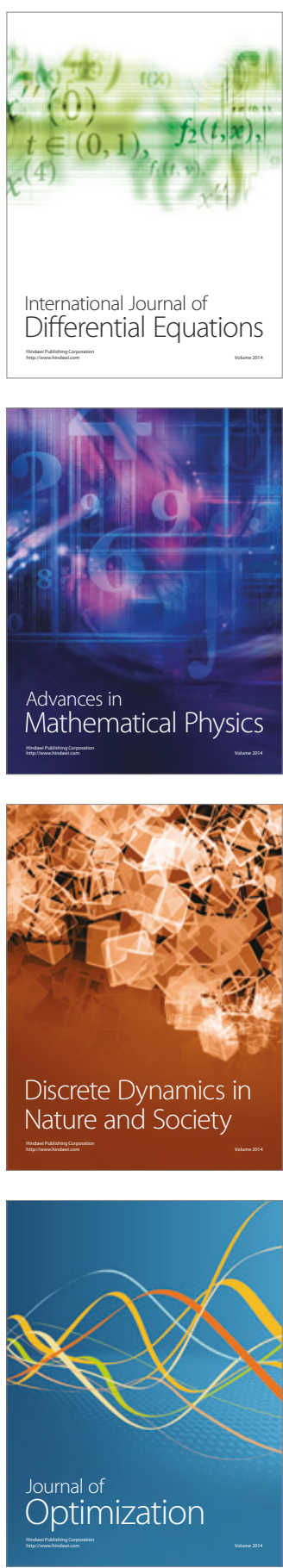